Abstract P192 Table 1 Functional respiratory imaging, body plethysmography and spirometry endpoints on Day 15 (end of treatment period)

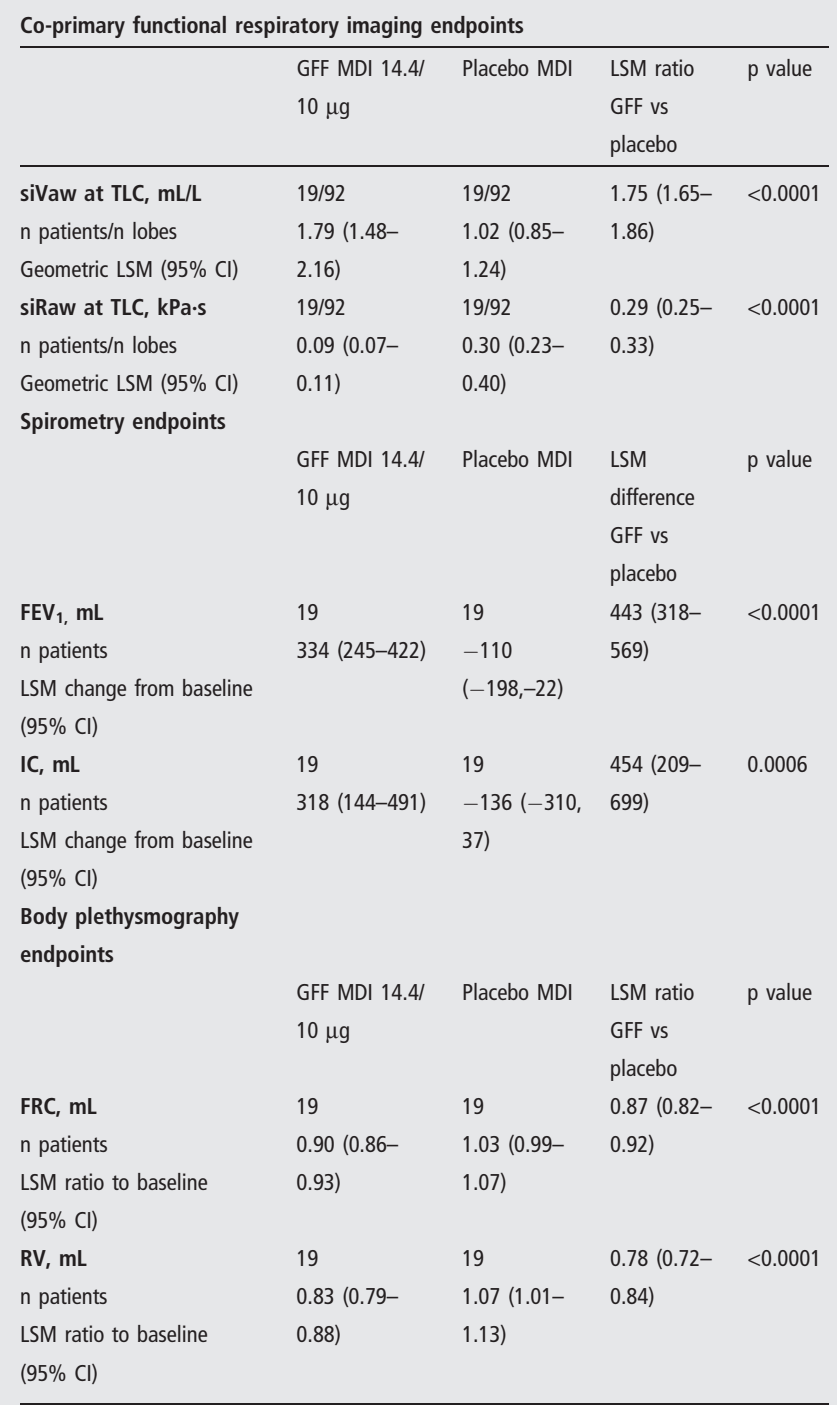

$\mathrm{Cl}$, confidence interval; $\mathrm{FEV}$, forced expiratory volume in $1 \mathrm{~s}$; FRC, functional residual capacity; GFF, glycopyrronium/formoterol fumarate dihydrate; IC, inspiratory capacity; LSM, least squares mean; $\mathrm{MDI}$, metered dose inhaler; RV, residual volume; siRaw, specific image-based airway resistance; siVaw, specific image-based airway volume; TLC, total lung capacity.

\section{P193 THE DEGREE OF LUNG DESTRUCTION WITH EMPHYSEMA ON QUANTITATIVE LUNG CT SCANS VERSUS SUBJECTIVE AND OBJECTIVE IMPAIRMENT IN PATIENTS WITH ADVANCED EMPHYSEMA REFERRED FOR VOLUME REDUCTION THERAPIES}

DT Betney, N Jarad. Bristol Royal Infirmary, Bristol, UK

\subsection{6/thoraxjnl-2017-210983.335}

Background Quantitative CT (QCT) scans of the lungs have been recently introduced for directing clinicians to the most appropriate lobes needing treatment with lung volume reduction (LVR) therapies. Changes in QCT have been considered as a key marker of procedure success. However, despite procedures aiming to improve quality of life and exercise tolerance, there has been no understanding if the degree of emphysema on QCTs correlates with the subjective and objective parameters used in patient selection for LVR.

Methods The pre-treatment QCT used was able to segment the lungs by tracing inter-lobar fissures thus providing data on the volume of each lobe. It was also able to digitally assess the proportion of emphysematous tissue area (defined by Hounsfield units of -910 or less) in each lobe. Utilising these two properties we calculated the volume of the lungs affected by emphysema by the summation of the emphysema volumes in all lobes. Values and percentage predicted of FEV1, residual volumes (RV) and gas transfer for carbon monoxide (TLco) were obtained from standard measurements; along with a 6 min walk distance (6'WD) and COPD assessment test (CAT) score. Spearman non-parametric correlation test was used to correlate emphysema volume with these parameters.

Results A total of 47 patients (19 female), mean age (SD) of 66.2 (8.9) years were included. Their mean (SD) FEV1 was $0.81 \mathrm{~L}(0.28)$. There was no correlation between the total emphysema volume and CAT score $(r=-0.21, p=0.2)$ or with 6'WD $(\mathrm{r}=-0.34, \mathrm{p}=0.054)$. Total emphysema volume and the value of RV were strongly correlated; $\mathrm{r}=0.68, \mathrm{p}<0.0001$. There was no correlation with FEV1 or TLco values. However, percentage of predicted values of lung function tests weakly correlated with total emphysema volume; for FEV1 $(\mathrm{r}=-0.36, \mathrm{p}=0.01)$, for $\mathrm{RV}(\mathrm{r}=0.34, \mathrm{p}=0.02)$ and for TLco $(\mathrm{r}=-0.32, \mathrm{p}=0.04)$.

Conclusion The lack of strong correlation between anatomical changes and lung function is probably due to changes in airway diameter (as well as tissue destruction) which is not captured by QCT. To add to that, the lack of correlation with 6'WD or CAT score is probably due to non-pulmonary factors affecting the values of these two measurements.

\section{P194 LOBAR PERFUSION UPTAKE SIGNIFICANTLY DIFFERS FROM LOBAR LUNG DESTRUCTION IN PATIENTS WITH ADVANCED EMPHYSEMA REFERRED FOR VOLUME REDUCTION THERAPIES}

DT Betney, N Jarad. University Hospital Bristol, Bristol, UK

\subsection{6/thoraxjnl-2017-210983.336}

Background Lung perfusion scan is widely undertaken as part of the assessment to select most affected lobes by emphysema prior to lung volume reduction (LVR) therapies by endo-bronchial valves, endo-bronchial coils and LVR surgery. More recently quantitative CT scans (QCT) have been introduced. QCTs quantify the degree of lung destruction by emphysema. To date there are no studies to evaluate whether lobar uptake of isotope by perfusion scan and areas of destruction on QCT's are closely correlated.

Methods Patients referred for LVR therapies at our hospital underwent perfusion scans using single-photon emission computerised tomography (SPECT) scans. The degree of uptake of isotope in each lobe is visually scored from $0-10$, 0 representing low lobar isotope uptake and 10 high uptake. The degree of lobar low attenuation area (emphysema) has been assessed by a QCT scan. This scan provided data on the proportion of each lobe with attenuation of -910 Hounsfield Units (HU) and -950 HU. Uptake on SPECT has been correlated with emphysema score on QCTs.

Results A total 47 patients are included (20 female). Their mean age 66.2 years, and mean FEV1 $30 \%$ of predicted 


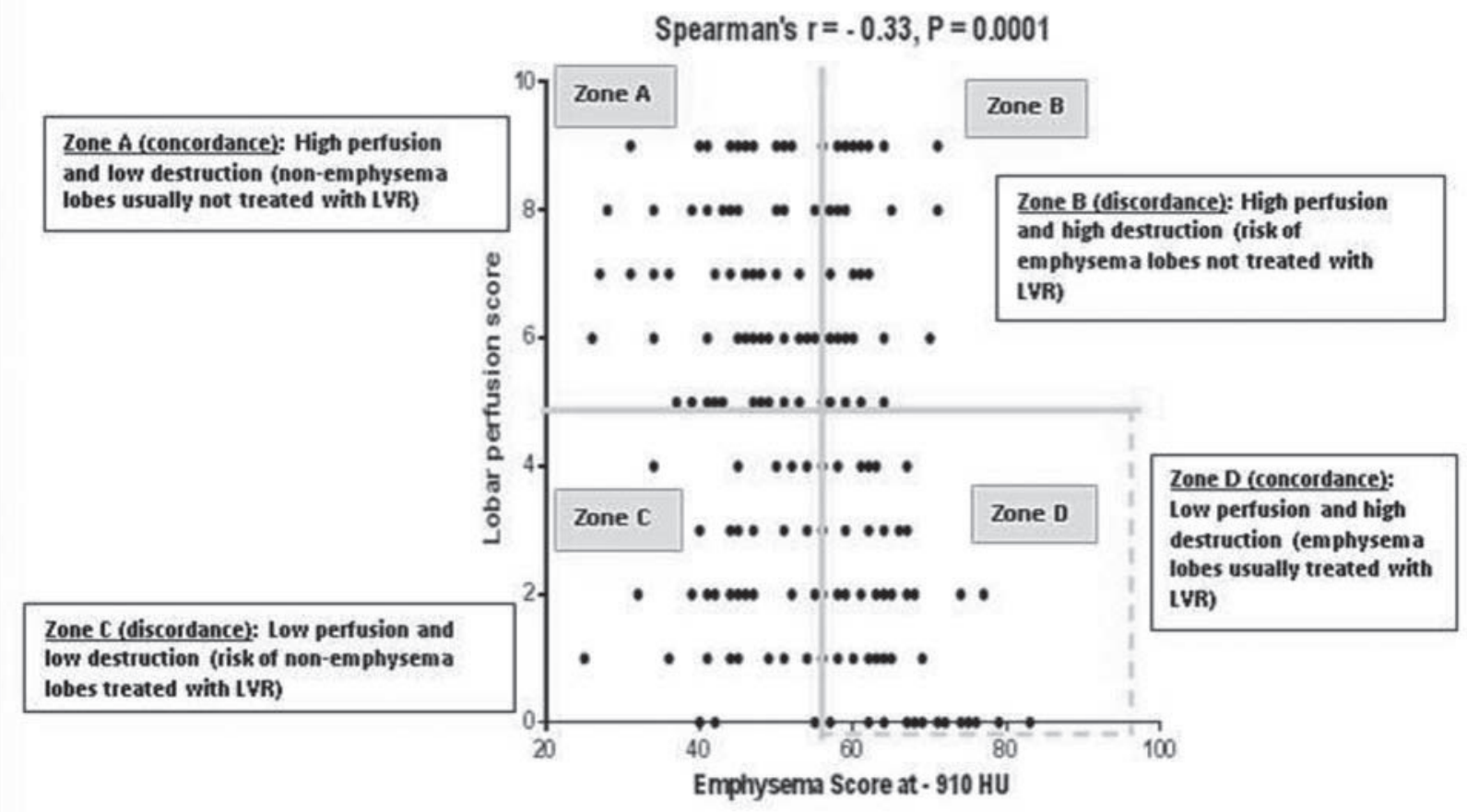

Graph1. Showing the concordanoe or discordance betveen lober perfusionand destruction based onermphyserrm sco re from QCT scan.

\section{Abstract P194 Figure 1}

values. Analysis by QCT and SPECT is available on 235 lung lobes. For all lung lobes, mean low attenuation at $-910 \mathrm{HU}$ was $53.7 \%$ and at $-950 \mathrm{HU}$ of $36.9 \%$. Median Score on SPECT was 5.3 points. A weak correlation between uptake score on SPECT and QCT scores; Spearman $\mathrm{r}=-0.33$, $\mathrm{p}<0.0001$ for emphysema area at $-910 \mathrm{HU}$ and $\mathrm{r}=-0.33$, $\mathrm{p}<0.001$ for emphysema area at -950 HU. Significant discordance is present between the two methods (graph.1) which could lead to either treating lobes with low perfusion but preserved lung tissue or not treating lobes with high perfusion but with significant emphysema.

Conclusion Despite the wide usage of perfusion scan to guide identification of lung lobes targeted for LVR, this study shows that this method needs to be interpreted with caution. QCT's should be relied upon to choose lobes needing treatment. Longitudinal analysis is needed to evaluate the outcome of treatment when the treated lobe was selected according to low perfusion.

\section{External influences on asthma}

\section{P195 "SYNDROME Z" IN THE ASTHMA POPULATION}

${ }^{1} \mathrm{~S}$ Davies, ${ }^{2} \mathrm{~N}$ Cachada, ${ }^{2} \mathrm{~S}$ Wharton, ${ }^{3} \mathrm{AM}$ Turner, ${ }^{1} \mathrm{~A}$ Mansur. ${ }^{1}$ Birmingham Regional Severe Asthma Service, Heartlands Hospital, Birmingham, UK; ${ }^{2}$ Birmingham Sleep Service, Heartlands Hospital, Birmingham, UK; ${ }^{3}$ Institute of Applied Health Research, University of Birmingham, Birmingham, UK

10.1136/thoraxjnl-2017-210983.337
Introduction Current literature demonstrates associations between asthma, obstructive sleep apnoea (OSA) and obesity. Syndrome $\mathrm{Z}$ is the occurrence of OSA with the metabolic syndrome, and the relevance of this condition in asthma populations remains unclear.

Methods 192 patients were recruited from a regional severe asthma service and associated respiratory clinics during January 2016-June 2017. 37 had a pre-existing diagnosis of OSA, 116 patients were screened regardless of symptoms, 39 patients with symptoms of OSA were included. Patients underwent an overnight limited channel sleep study and bioelectrical impedance measurements. The groups were split into OSA and noOSA to compare metabolic profile, associated co-morbidities and body fat composition. Data were analysed using MedCalc version 15.

Results 192 patients with asthma (137 females, 55 males), 173 (90\%) had severe asthma, 19 (10\%) had non-severe asthma. $37(19.3 \%)$ had pre-existing OSA, 26 of which required Continuous Positive Airway Pressure(CPAP). A total of 97 (51\%) had OSA, 58 (30\%) had OSA excluded. The OSA group had significantly higher mean Body Mass Index (BMI) $(34.8$ \pm 8.2 versus no-OSA group $28.1 \pm 6.0, \mathrm{p}<0.001)$, body fat $\%$ $(38.2 \% \pm 11 \%$ versus no-OSA group $32.2 \% \pm 12 \%, \mathrm{p}=0.002)$, visceral fat rating $(12.8 \pm 5.1$ versus no-OSA group $7.4 \pm 4.1$, $\mathrm{p}<0.001)$ and mean metabolic age $(59.5 \pm 12.8$ years versus no-OSA $44.4 \pm 16.7$ years, $\mathrm{p}<0.001$ ). The OSA group also had significantly higher rates of diabetes (OSA $0.25 \pm 0.47$, no-OSA $0.06 \pm 0.23, \mathrm{p}=0.005$ ), hypercholesterolaemia (OSA 51/132 (38.6\%), no-OSA 9/53 (28.6\%), $\mathrm{p}=0.0046)$ and hypertension (OSA 50/132 (37.9\%), no-OSA 6/53 (10.7\%), p=0.0004). 